\title{
Inflammatory Factors as Potential Markers of Early Neurological Deterioration in Acute Ischemic Stroke Patients Receiving Endovascular Therapy - The AISRNA Study
}

\author{
Qi-Wen Deng $\mathbb{I}^{1, *}$ \\ Shi Huang ${ }^{l, *}$ \\ Shuo $\mathrm{Li}^{2, *}$ \\ Qian Zhai ${ }^{1}$ \\ Qing Zhang' \\ Zhen-Jie Wang' \\ Wen-Xia Chen' \\ Huiling Sun ${ }^{3}$ \\ Min Lu' \\ Junshan Zhou'
}

'Department of Neurology, Nanjing First Hospital, Nanjing Medical University, Nanjing, 210006, People's Republic of China; ${ }^{2}$ Department of Neurology, Affiliated ZhongDa Hospital, School of Medicine, Southeast University, Nanjing, 210009, People's Republic of China;

${ }^{3}$ General Clinical Research Center, Nanjing First Hospital, Nanjing Medical University, Nanjing, 210006, People's Republic of China

*These authors contributed equally to this work

\begin{abstract}
Background and Purpose: This study aimed to explore several peripheral blood-based markers related to the inflammatory response in a total of 210 patients with acute ischemic stroke (AIS) caused by large artery occlusion in the anterior circulation who received endovascular therapy (EVT) from an observational study of clinical significance of circulating non-coding RNA in acute ischemic stroke (AISRNA).
\end{abstract}

Methods: We collected baseline characteristics of 210 AIS patients participating in an observational acute stroke cohort: the AISRNA study. The following inflammatory factors were measured in these participants: interleukin-2 [IL-2], IL-4, IL-6, IL-10, tumor necrosis factor- $\alpha$ [TNF- $\alpha]$, and interferon- $\gamma[$ IFN- $\gamma]$. The National Institute of Health Stroke Scale score increase of $\geq 4$ within 24 hours after EVT defined as early neurological deterioration (END).

Results: Compared with patients without END, patients with END had a higher incidence of atrial fibrillation $(P=0.012)$, and also had higher levels of IL-6 and IL-10 $(P<0.01)$. Furthermore, we found that the area under the curves (AUCs) of IL- 6 and IL-10 for predicting END were $0.768(0.697-0.829)$, and $0.647(0.570-0.719)$, respectively. Adjusting for age, sex, and atrial fibrillation, the odds ratios (ORs; $95 \%$ confidence interval) for incident END for IL-6 and IL-10 were 1.98 (1.05-6.69) and 1.18 (1.04-1.33), respectively. Additionally, we found significant changes over time in the expression levels of IL-4, IL-6, and IL-10 in patients with END compared with patients without END $(P<0.05)$.

Conclusion: IL-6 and IL-10 levels at admission may be potential markers of END after EVT, and the time course of IL-4, IL-6, and IL-10 is correlated with stroke progression. Further larger studies are needed to confirm the current findings.

Trial Registration: ClinicalTrials.gov NCT04175691. Registered November 21, 2019, https://www.clinicaltrials.gov/ct2/show/NCT04175691

Keywords: acute ischemic stroke, early neurological deterioration, endovascular therapy, inflammatory factors, IL-6

\section{Introduction}

Stroke is commonly considered to be a major cause of disability and mortality in adults. Although the early advent of endovascular therapy (EVT) has improved the clinical outcomes of acute ischemic stroke (AIS), over $50 \%$ of patients still suffer from disabilities and deficits, which may be the result of neurological and medical complications. ${ }^{1}$ The precise prediction of clinical outcomes during the acute phase of
Correspondence: Junshan Zhou; Min Lu Department of Neurology, Nanjing First Hospital, Nanjing Medical University, 210006, People's Republic of China Email zhjsh333@126.com; luminnfhsjk@।26.com 
ischemic stroke after EVT remains challenging. While our previous studies have found that demographics and several clinical characteristics are associated with prognosis after acute ischemic stroke, ${ }^{2-4}$ the accuracy of prediction remains limited, especially during the acute phase of IS. ${ }^{5}$ Therefore, the development of precise scores to predict the prognosis in the acute stage may benefit from the identification of individual biomarkers.

A correlation between stroke and the acute inflammatory response can be found in various stages of acute infection. Accumulating evidence has indicated that stroke induces a rapid immunodepression through the autonomic nervous system. ${ }^{6,7}$ Immune factors, including cytokines, are indicative of stroke-associated infection and are associated with clinical outcome after AIS, ${ }^{8,9}$ which may be attributed to dynamic changes in the secretion of inflammatory cytokines in the central and peripheral immune responses affecting the progression of AIS. ${ }^{10}$ These inflammatory cytokines are mostly produced by activated peripheral immune cells and resident microglial cells. ${ }^{11}$ Mounting evidence showed that Th2 type cytokines including interleukin-10 (IL-10) and IL-4 are involved in the repair of brain injury and the inhibition of stroke-associated inflammation. ${ }^{12,13}$ Unexpectedly, IL-6, well known for a Th1 type cytokine presenting the proinflammatory effect, also represents neurotrophic and regenerative capabilities after cerebral infarction. ${ }^{14}$ Therefore, these inflammatory cytokines may be associated with stroke pathogenesis and outcome. Previous studies have revealed the functions of inflammatory cytokines in an acute phase of ischemic stroke. ${ }^{9,15}$ However, the link between inflammatory cytokines and stroke progression remains unclear in AIS patients receiving EVT.

In the present study, we enrolled a total of 210 patients who received EVT from the Clinical Significance of Circulating Non-coding RNA in Acute Ischemic Stroke (AISRNA) study to investigate a range of inflammatory factors (interleukin-2 [IL-2], IL-4, IL-6, IL-10, tumor necrosis factor- $\alpha[\mathrm{TNF}-\alpha]$, and interferon- $\gamma[\mathrm{IFN}-\gamma]$ ) and early neurological deterioration (END) after stroke caused by large artery occlusion in the anterior circulation. Additionally, the influence of END after EVT on time course of inflammation-related biomarkers was further explored.

\section{Materials and Methods}

\section{Study Population}

All subjects provided informed consent. In the present study, we enrolled consecutive patients with AIS patients caused by large artery occlusion in the anterior circulation who received EVT. Data from a total of 210 AIS patients who received EVT were prospectively collected from an observational study of the AISRNA study (www.clinical trials.gov, NCT04175691) to investigate the association of inflammatory factors at admission and their dynamic changes with END for 7 days. The clinical characteristics of the patients are summarized in Table 1. All the patients were from Nanjing First Hospital, Nanjing Medical University which is a stroke center affiliated with China Stroke Association. Eligible patients were enrolled in the present study if they met the following inclusion criteria: (1) AIS patients who received EVT and (2) patients with anterior circulation occlusion. Patients were excluded from the study if they met the following exclusion criteria: (1) patients with intracranial hemorrhage; (2) patients with infection on admission; (3) patients treated with antibiotics or immunodepression medical therapy within the past 4 weeks; (4) patients under 18 years of age; (5) patients with a history of a malignant tumor; and (6) patients who died within 7 days. Clinical outcomes (modified Rankin Scale [mRS]) were further investigated after 3 months. Eligible participants were followed up after 3 months via telephone and contact in an outpatient clinic. Our study protocol was approved by the Nanjing Medical University Ethics Committee and complied with the Declaration of Helsinki.

\section{Clinical Data}

The data collected included demographics, medical history, stroke severity at admission and $24 \mathrm{~h}$ later, stroke etiology, laboratory parameters, modified Thrombolysis in Cerebral Infarction (mTICI) Score, and time of onset to door, groin puncture, and reperfusion. Several data definitions were used in the present study: the National Institute of Health Stroke Scale (NIHSS) was used to assess stroke severity; the delta NIHSS scale (delta NIHSS 0-24h) = NIHSS on admission NIHSS after $24 \mathrm{~h}$; early neurological deterioration (END) was defined as $\triangle \mathrm{NIHSS} \geq 4$ points; ${ }^{16}$ and stroke etiology was determined according to the Trial of Org 10172 in acute stroke treatment (TOAST) criteria. $^{17}$

\section{Inflammation-Related Biomarkers}

Blood samples were collected within $24 \mathrm{~h}$ after EVT (day 1) and repeated on days 2, 3 and 7. Plasma samples were centrifuged (2000rpm, $4^{\circ} \mathrm{C}, 20 \mathrm{~min}$ ) and stored at $-80^{\circ} \mathrm{C}$. The plasma concentrations of IL-2, IL-4, IL-6, IL-10, TNF- $\alpha$, and IFN- $\gamma$ were determined with the Navios flow cytometer 
Table I Baseline Characteristics of the Eligible Patients Stratified by END

\begin{tabular}{|c|c|c|c|c|}
\hline Variable & Total & Non-END & END & p-value \\
\hline n (\%) & $n=210$ & $n=187$ & $n=23$ & \\
\hline \multicolumn{5}{|l|}{ Demographics } \\
\hline Sex (female) & $79(37.6)$ & $72(38.5)$ & $7(30.4)$ & $0.45 I$ \\
\hline Age (years) & $67.98 \pm \mid 2.1$ & $67.34 \pm 12.7$ & $70.61 \pm 9.6$ & 0.528 \\
\hline BMI & $24.7 I \pm 3.3$ & $24.28 \pm 3.6$ & $25.60 \pm 3.8$ & 0.132 \\
\hline \multicolumn{5}{|l|}{ Medical history } \\
\hline Hypertension & $142(67.6)$ & $129(69.0)$ & $13(56.5)$ & 0.228 \\
\hline DM & $45(21.4)$ & $39(20.9)$ & $6(26.1)$ & 0.564 \\
\hline CAD & $29(13.8)$ & $26(13.9)$ & $3(13.0)$ & 0.910 \\
\hline $\mathrm{AF}$ & $4 \mid(19.5)$ & $32(17.1)$ & $9(39.1)$ & 0.012 \\
\hline IS or TIA & $28(13.3)$ & $22(11.8)$ & $6(26.1)$ & 0.057 \\
\hline \multicolumn{5}{|l|}{ Stroke etiology ${ }^{\mathrm{a}}$} \\
\hline LAA & $72(34.3)$ & $63(33.7)$ & $9(39.1)$ & 0.407 \\
\hline Cardioembolism & $98(46.7)$ & $86(46.0)$ & $12(52.2)$ & \\
\hline Others & $40(191.0)$ & $38(20.3)$ & $2(8.7)$ & \\
\hline NIHSS on admission & $14(5-22)$ & $13(||-2 \mid .5)$ & $17(6.5-29)$ & 0.074 \\
\hline NIHSS after $24 \mathrm{~h}$ & II (4-20) & $10(3.5-19)$ & $30(18-34)$ & $<0.001$ \\
\hline 90-day mRS & $2(0-4)$ & I (0-3) & $4(2-5)$ & $<0.001$ \\
\hline IVT & $88(41.9)$ & 78 (4I.7) & $10(43.5)$ & $0.87 I$ \\
\hline \multicolumn{5}{|l|}{ Interval time, min, median (IQR) } \\
\hline Onset to groin puncture & $106(81.5-144)$ & $104(82-136)$ & $111(73-160.5)$ & 0.540 \\
\hline Onset to reperfusion & $206.5(13 \mid-272)$ & $195(136-218)$ & $227(128.5-302.5)$ & 0.585 \\
\hline EVT to first blood sampling ${ }^{b}$ & $815(564-1045)$ & $772(564-1000)$ & $847.5(550.5-1087)$ & 0.425 \\
\hline $\mathrm{mTICI}(2 \mathrm{~b}-3)$ & $184(87.6)$ & $165(88.2)$ & $19(82.6)$ & 0.439 \\
\hline Passes of retriever $(<3)$ & $142(67.6)$ & $128(68.4)$ & $14(60.9)$ & 0.464 \\
\hline \multicolumn{5}{|l|}{ Inflammatory factors on admission } \\
\hline IL-2 (pg/mL) & $2.52 \pm 1.2$ & $2.55 \pm 1.1$ & $2.64 \pm 1.4$ & 0.526 \\
\hline IL-4 (pg/mL) & $3.12 \pm 1.7$ & $2.89 \pm 1.7$ & $3.39 \pm 1.6$ & 0.681 \\
\hline IL-6 (pg/mL) & I $3.54(6.81-30.60)$ & $10.08(6.62-25.68)$ & $28.04(15.72-107.06)$ & 0.002 \\
\hline IL-10 (pg/mL) & $5.16(4.02-7.26)$ & $5.05(4.11-7.50)$ & $6.02(3.96-26.45)$ & $<0.001$ \\
\hline TNF- $\alpha(\mathrm{pg} / \mathrm{mL})$ & $2.18(1.24-3.11)$ & $2.12(1.31-2.85)$ & $2.30(1.20-3.96)$ & 0.206 \\
\hline INF- $\gamma(\mathrm{pg} / \mathrm{mL})$ & $2.42(1.61-3.24)$ & $2.35(1.75-3.18)$ & $2.23(0.92-3.30)$ & 0.227 \\
\hline \multicolumn{5}{|l|}{ Laboratory characteristics } \\
\hline hs-CRP $(\mu \mathrm{g} / \mathrm{mL})$ & $4.93(4.32-10.76)$ & $4.86(4.20-9.08)$ & $5.12(4.43-11.99)$ & 0.197 \\
\hline $\mathrm{HbAlc}$ & $5.82(5.46-6.50)$ & $5.81(5.44-5.82)$ & $6.01(5.51-6.66)$ & 0.442 \\
\hline Fasting glucose (mmol/L) & $6.83(5.5 \mathrm{I}-8.59)$ & $6.70(5.46-8.52)$ & $6.95(6.04-8.68)$ & 0.567 \\
\hline Creatinine $(\mu \mathrm{mol} / \mathrm{L})$ & $67.5(56.4-90.8)$ & $66.9(56.0-90.5)$ & $69.3(56.8-95.4)$ & 0.386 \\
\hline LDL-C (mmol/L) & $2.82 \pm 1.1$ & $2.80 \pm 1.0$ & $2.35 \pm 0.8$ & 0.259 \\
\hline
\end{tabular}

Notes: $\triangle$ NIHSS 0-24h was defined as the delta NIHSS scale (delta NIHSS 0-24h) $=$ NIHSS on admission - NIHSS after 24 hours. Continuous variables of abnormal distribution are expressed as medians (interquartile range), and normally distributed variables as mean \pm standard deviation (SD). Categorical variables are expressed as frequency and percentage. according to the modified TOAST classification. ${ }^{\text {b }}$ Defined as time between endovascular treatment and first blood sampling to be measured levels of inflammatory factors.

Abbreviations: END, early neurological deterioration; EVT, endovascular treatment; mRS, modified Rankin scale; IL, interleukin; TNF, tumor necrosis factor; INF, interferon; BMI, body mass index; DM, diabetes mellitus; CAD, coronary artery disease; AF, atrial fibrillation; IS, ischemic stroke; TIA, transient ischemic attack; IQR, interquartile range; mTICl, modified Thrombolysis in Cerebral Infarction Score; NIHSS, National Institute of Health Stroke Scale; LAA, large artery atherosclerosis; LDL-C, low-density lipoprotein cholesterol; HDL-C, high-density lipoprotein cholesterol; hs-CRP, high-sensitivity C-reactive protein; IVT, intravenous thrombolysis. 
(Beckman Coulter, California, USA). The upper limit of detection for these factors was $2500 \mathrm{pg} / \mathrm{mL}$.

\section{Statistical Analysis}

Categorical variables are expressed as frequency and percentage. Continuous variables of an abnormal distribution are expressed as medians (interquartile range [IQR]), and normally distributed variables are expressed as the mean \pm standard deviation (SD). The $t$-test, one-way ANOVA and Mann-Whitney $U$-tests were used to analyze continuous variables if necessary. Bivariate correlation between delta NIHSS score, 90-day mRS and inflammatory factors was analyzed by the Spearman correlation. Variables from significant factors of the univariable logistic regression analysis were considered in the multivariate logistic regression model to obtain independent factors. Discriminatory capacities of inflammatory factors were assessed by receiver operating characteristic (ROC) curve analyses and area under the curve (AUC). The significance threshold was set at less than 0.05 .

\section{Results}

Of the 1236 patients screened from the AISRNA study between November 2019 and February 2021, a total of 210 participants (79 female; mean age, $67.98 \pm 12.1$ years) met the inclusion criteria (Figure 1). A total of 1026 patients were excluded according to the following criteria: lack of
EVT $(n=960)$, posterior circulation occlusion $(n=15)$, died within 7 days $(n=8)$, preexisting dysphagia $(n=7)$, intracranial hemorrhage $(n=8)$, infection on admission $(n=10)$, antibiotic or immunodepression therapy within the past 4 weeks $(n=6)$, and no blood samples $(n=12)$. The baseline characteristics of the eligible patients are summarized in Table 1. Of the 210 patients included, 88 (41.9\%) received intravenous thrombolysis.

A total of $23(11.0 \%)$ patients suffered END after EVT. The association between inflammatory factors at admission and incidence of END is shown in Table 1. The NIHSS on admission was not significantly different between the groups $(P=0.074)$. However, compared with non-END, NIHSS after $24 \mathrm{~h}$ and 90-day mRS were significantly higher in patients with END $(P<0.001)$. We found that END was associated with increased IL-6 and IL-10 levels having a higher proportion of atrial fibrillation $(P=0.012)$. As the delta NIHSS was considered END, we performed a correlation analysis to investigate the correlation between inflammatory factors and delta NIHSS 0-24 $\mathrm{h}$. The results showed that the delta NIHSS was correlated with the expression of IL-6 $(\mathrm{r}=0.260, P=0.016)$ and IL-10 $(\mathrm{r}=-0.238, \quad P=0.028) \quad$ (Figure $\mathrm{S} 1 \mathrm{~B}$ and $\underline{\mathrm{S} 1 \mathrm{C}})$, but a correlation was not observed with the expression of IL2 , IL-4, INF- $\gamma$, and TNF- $\alpha(P>0.05$, Figure S1A, and $\underline{\mathrm{S} 1 \mathrm{D}-\mathrm{F})}$. Furthermore, we also performed a correlation analysis to explore the association between inflammatory

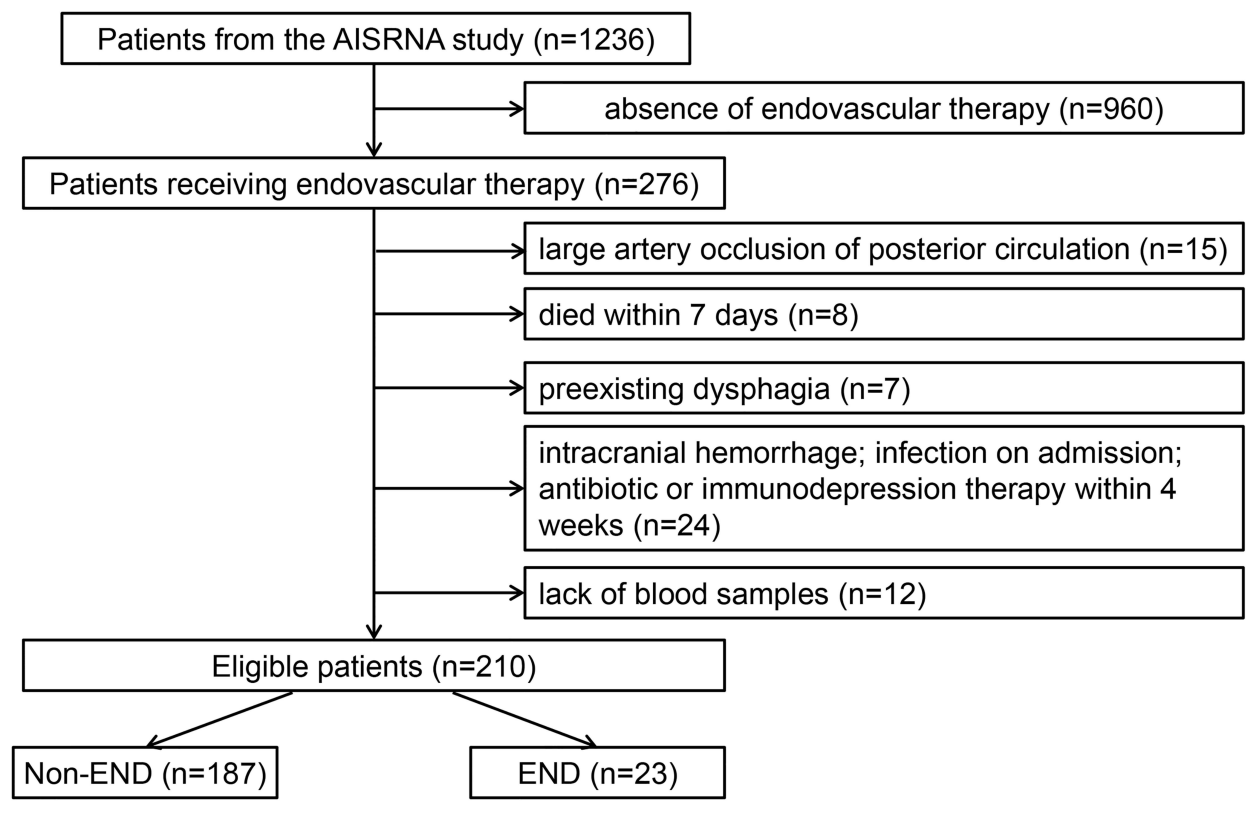

Figure I Flowchart of the study patients to illustrate study screening, recruitment, and follow-up. Abbreviation: END, early neurological deterioration. 
factors and 90-day mRS. The findings suggested that serum concentration of IL-6 was significantly associated with 90-day mRS after EVT $(\mathrm{r}=0.171, P=0.025$, Figure $\underline{\mathrm{S} 2 \mathrm{~B}}$ ), but not IL-2, IL-4, IL-10, INF- $\gamma$, and $\mathrm{TNF}-\alpha$

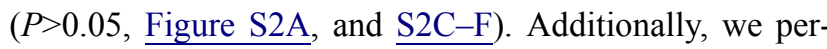
formed an ROC curve analysis to explore the predictive powers of these factors. We observed that the AUCs of IL6 and IL-10 for predicting END were 0.768 (0.697-0.829), and $0.647(0.570-0.719)$, respectively (Figure 2$)$, revealing that IL-6 outperformed IL-10 in predicting END $(P<0.05)$.

Given that association of inflammatory factors with END, we conducted a binary logistic regression to analyze the impact of inflammatory factors on END. The results of the univariate analyses showed that the IL-6 and IL-10 levels were prognostic factors for END, and the findings remained stable after adjustment (Table 2).

To further study the clinical utility of inflammatory factors after EVT, we analyzed the time courses of the inflammatory factors within 7 days for patients with and without END (Figure 3). A total of 97 blood samples were collected for day 1 , day 2 , day 3 and 7 among these patients. We found significant changes over time in the expression levels of IL-4, IL-6, and IL-10 in patients with END compared without END (Figure $3 \mathrm{~A}-\mathrm{C}, P<0.05$ ), and the IL-6 levels were obviously increased from days 1 to 7 after EVT (Figure 3B, $P<0.05$ ). Furthermore, we observed IL-4 and IL-10 peak levels at day 2, and then rapidly decreased at day 3 (Figure $3 \mathrm{~A}$ and $\mathrm{C}$ ). However, No significant change over time was found in the expression levels of IL-2 (Figure 3D), TNF- $\alpha$ (Figure 3E), and INF- $\gamma$ (Figure 3F) between patients with END and patients without END $(P>0.05)$.

\section{Discussion}

This study of EVT participants from the AISRNA study showed a strong association of IL-6 and IL-10 levels at admission with risk of END. Our study suggests that patients with increased IL-6 and IL-10 levels had a higher risk of developing post-EVT END. By contrast, there was no correlation of baseline levels of IL-2, Il-4, INF- $\gamma$, and TNF- $\alpha$ with END. The predictive power of IL6 for END was superior to that of other inflammatory

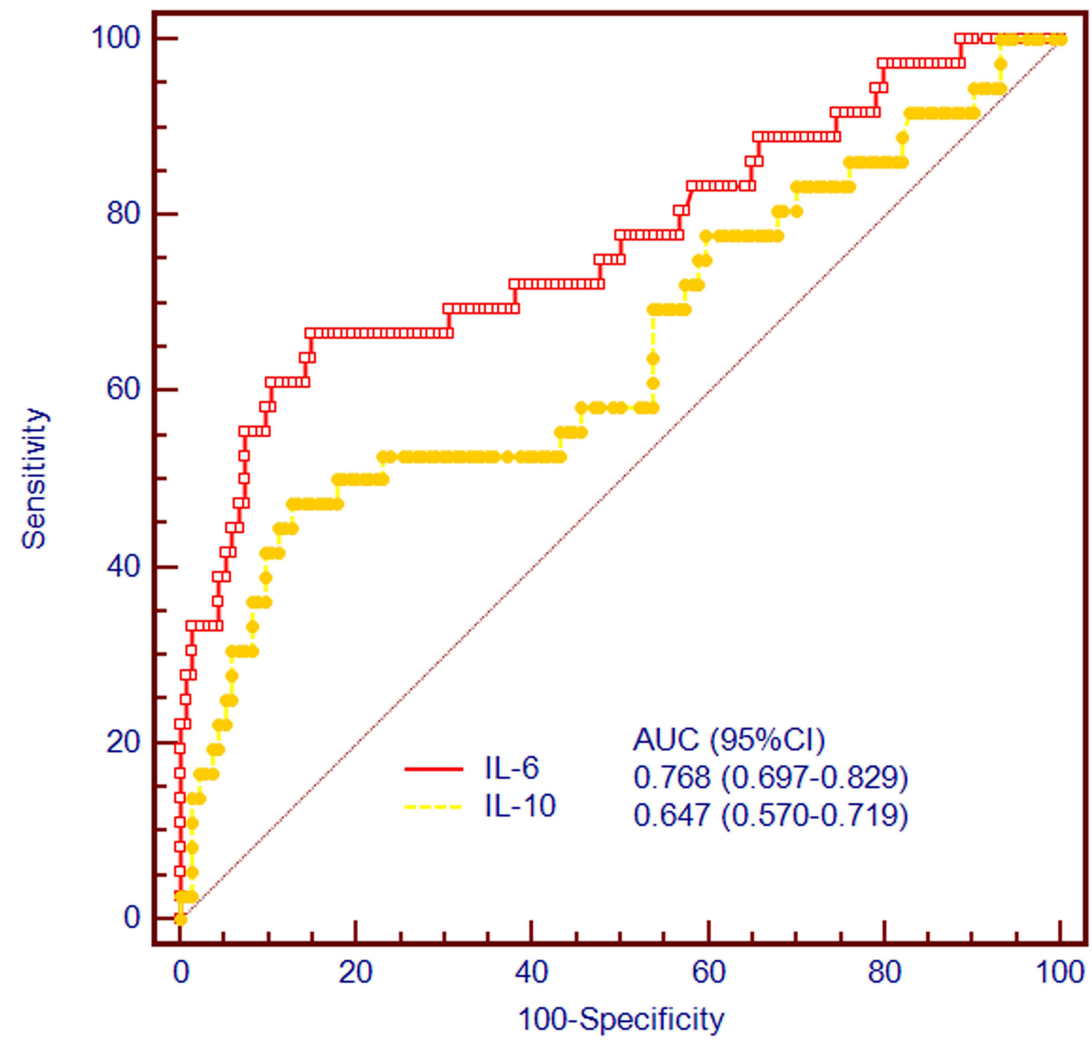

Figure 2 Discriminatory capacities of IL-6 and IL-I0 for END after EVT. The area under the curves of IL-6 and IL-I0 for predicting END were 0.79I (0.689-0.87I), and 0.564 (0.452-0.67I), respectively $(P<0.00 \mathrm{I})$.

Abbreviations: END, early neurological deterioration; EVT, endovascular therapy. 
Table 2 Logistic Regression Analyses for Inflammatory Factors Associated with END

\begin{tabular}{|c|c|c|c|}
\hline Variable & Model $^{\mathrm{a}}$ & OR (95\% Confidence Interval) & p-value \\
\hline \multirow[t]{3}{*}{ IL-2 } & 1 & $1.14(0.62-2.14)$ & 0.512 \\
\hline & 2 & $\ldots$ & $\ldots$ \\
\hline & 3 & $\ldots$ & $\ldots$ \\
\hline \multirow[t]{3}{*}{ IL-4 } & 1 & $1.03(1.00-1.04)$ & 0.052 \\
\hline & 2 & $\ldots$ & $\ldots$ \\
\hline & 3 & $\ldots$ & $\ldots$ \\
\hline \multirow[t]{3}{*}{ IL-6 } & 1 & $1.68(1.03-2.64)$ & 0.012 \\
\hline & 2 & $2.04(1.10-6.62)$ & 0.016 \\
\hline & 3 & $1.98(1.05-6.69)$ & 0.035 \\
\hline \multirow[t]{3}{*}{ IL-I0 } & 1 & $1.10(1.05-1.30)$ & 0.023 \\
\hline & 2 & $1.15(1.05-1.32)$ & 0.018 \\
\hline & 3 & $1.18(1.04-1.33)$ & 0.022 \\
\hline \multirow[t]{3}{*}{ TNF } & 1 & $1.33(0.74-1.89)$ & 0.658 \\
\hline & 2 & $\ldots$ & $\ldots$ \\
\hline & 3 & $\ldots$ & $\ldots$ \\
\hline \multirow[t]{3}{*}{ INF } & 1 & $0.94(0.56-1.87)$ & 0.789 \\
\hline & 2 & $\ldots$ & $\ldots$ \\
\hline & 3 & $\ldots$ & $\ldots$ \\
\hline
\end{tabular}

Note: ${ }^{a}$ Model I: unadjusted; model 2: adjusted for age and sex; model 3 adjusted for model 2 with atrial fibrillation.

Abbreviations: END, early neurological deterioration; IL, interleukin; TNF, tumor necrosis factor; INF, interferon; OR, odds ratio.

factors. Additionally, the IL-4, IL-6, and IL-10 levels presented a marked rising trend in patients with END.

Inflammation is a hallmark of stroke etiology and progression. Poststroke inflammation is considered a requisite pathological process involved in ischemic brain injury. ${ }^{18}$ A series of detrimental complications occur and the bloodbrain barrier (BBB), damaged after the initial brain injury, is crossed by activated peripheral immune cells including monocytes and T cells. ${ }^{19}$ Furthermore, poststroke inflammatory response is associated with stroke severity at admission as determined by NIHSS. ${ }^{20}$ Additionally, patients who suffered END were more susceptible to a poor functional outcome after 90 days. ${ }^{21}$ However, the association between poststroke inflammatory response and END remains uncertain. Therefore, we performed a prospective study from the AISRNA study to further investigate the influence of inflammatory factors on END after stroke.

The biological function of IL-6 in AIS remains controversial. ${ }^{22}$ IL-6 is considered as multipotent functions involving in brain damage and nerve regeneration. ${ }^{23}$ Firstly, IL-6 would enhance the brain injury and weaken the proliferation of neural stem cells in the acute phase of ischemic stroke. Secondly, IL-6 could inhibit collagen deposition to protect glial cells and repair brain injury in the subacute phage of cerebral ischemia. ${ }^{24} \mathrm{~A}$ previous study has demonstrated that the IL-6 levels are increased in peripheral blood samples during the first 7 days after stroke onset. ${ }^{25}$ For example, increased IL-6 is associated with the infarct size and stroke severity at admission ${ }^{12,26}$ as well as risk of incident stroke, ${ }^{27}$ but another study reported the opposite, that early levels of IL-6, as a neuroprotective factor, are inversely correlated with lesion size and functional outcome. ${ }^{28}$ Our findings also suggest that increased IL-6 was associated with risk of END after EVT. Thus, this controversial phenomenon needs to be further investigated. However, there is less agreement on the time point of the peak of IL-6 levels. Some studies reported IL-6 peak levels at day $3,{ }^{29,30}$ which is similar to our findings. Others describe high levels of IL-6 that ranged from a few hours until one day or a week after stroke. ${ }^{28,31,32}$

IL-10 is a major anti-inflammatory cytokine that is secreted from monocytes and $\mathrm{T}$ cells, suggesting its participation in a plethora of immunomodulatory functions. High expression of IL-10 promotes glial and neuronal cell survival and weakens inflammatory responses in the brain. ${ }^{33}$ In a permanent middle cerebral artery occlusion model, IL-10 suppresses proinflammatory molecules and reduces infarct volume. ${ }^{34}$ Several investigators also reported that IL-10 may serve a neuroprotective role and predict clinical outcome after stroke. ${ }^{12,35,36}$ However, other studies have suggested that IL-10 is a marker of incident stroke. ${ }^{37,38}$ Our previous studies from experimental rats and human stroke patients observed that IL-10 is positively associated with stroke risk. ${ }^{39,40}$ In the present study, we also reported a positive correlation between IL10 and END after EVT, suggesting that increased IL-10 levels at admission were associated with END after EVT. We observed IL-10 peak levels at day 2, and patients with END had decreased IL-10 levels compared with those with non-END at 7 days. This phenomenon may be due to a stress response of IL-10 regarding END, which leads to high levels of IL-10, but its levels were subsequently decreased after day 2. Therefore, IL-10 may be involved in the process of END, and its molecular mechanisms remain to be explored in further studies.

IL-4, an anti-inflammatory cytokine, can drive the differentiation of Th2 cell, which play beneficial roles in inhibiting poststroke inflammation, repairing damaged brain tissues and inducing neurotrophic factors in astrocytes. ${ }^{41,42}$ A previous study has demonstrated that IL-4 is significantly correlated with stroke severity and functional outcome in 

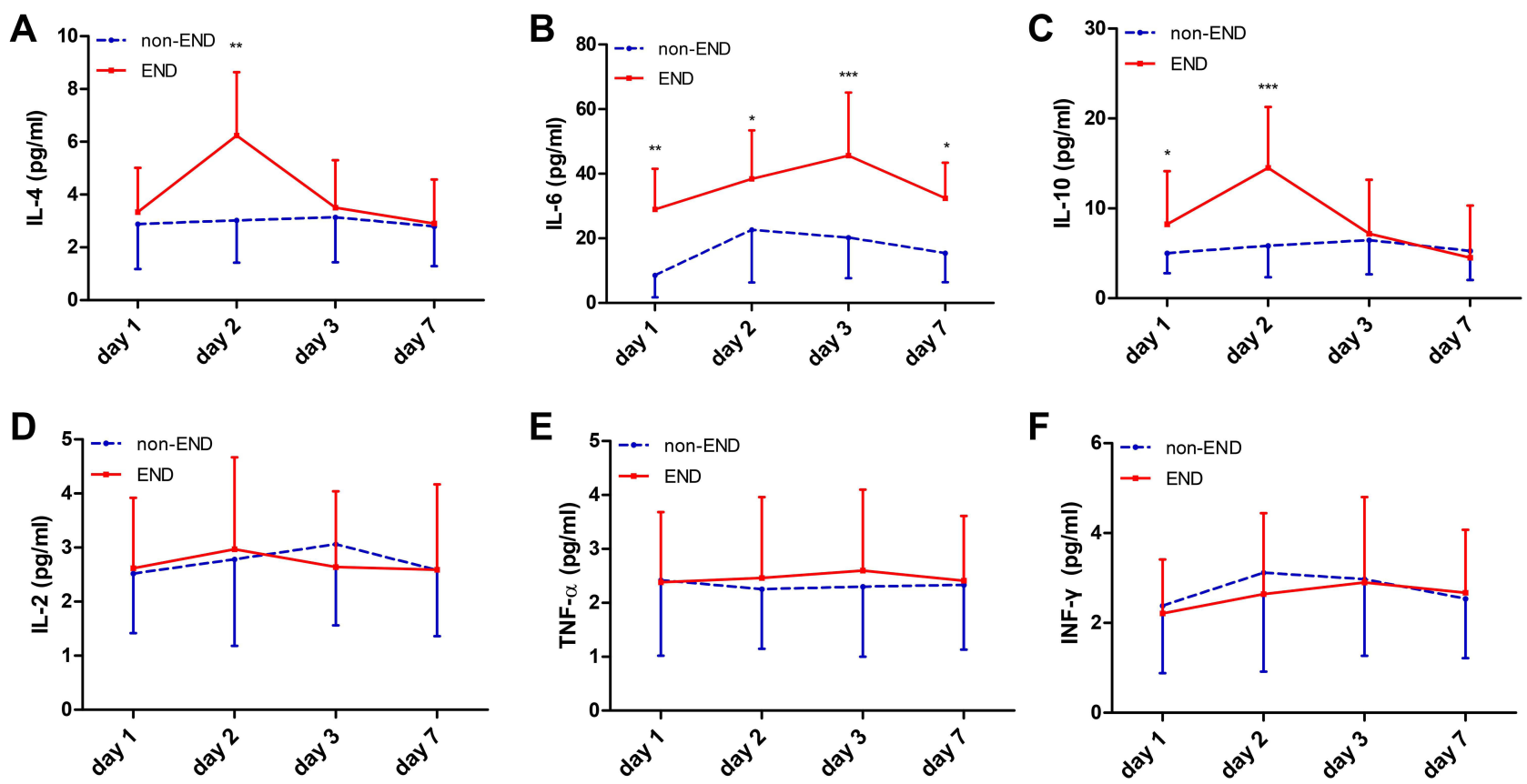

Figure 3 Time course of inflammatory factors after stroke onset (days I to 7). Significant changes over time were observed in the expression levels of IL-4, IL-6, and IL-I0 in patients with END compared with patients without END ( $P<0.05)$. IL-4 levels peaked at day 2 and then rapidly decreased at day 3 (A). IL-6 levels were obviously increased from days I to 7 after EVT (B). IL-10 (C) levels also peaked at day 2 and then rapidly decreased at day 3. No significant change over time was found in the expression levels of IL-2 (D), TNF- $\alpha(\mathbf{E})$, and INF- $\gamma(\mathbf{F})$ between patients with END and patients without END $(P>0.05)$. $* P<0.05, * * P<0.01$, $* * * P<0.001$.

Abbreviation: END, early neurological deterioration.

AIS patients. ${ }^{12}$ However, our results showed no association of IL-4 with END after EVT, but IL-4 levels were increased in patients with END at day 2 and then rapidly decreased at day 3. At present, we are also investigating the molecular mechanisms of IL-4 and IL-10 regarding dynamic change in stroke progression.

Several limitations should be acknowledged in the study. First, this study included a small number of patients from a single center. We are seeking for subcenters to complete the AISRNA study. Second, we did not analyze the association between the initial infarct burden and END after EVT. Third, although the incidence of atrial fibrillation in END patients was higher than those in non-END patients, the effect of inflammatory factors on END remained stable after adjustment for atrial fibrillation. Therefore, a randomized controlled study is urgent to confirm this finding. Finally, molecular mechanisms regarding dynamic changes of these inflammatory factors should be further explored in patients undergoing EVT.

In summary, this study illustrates the correlation of inflammatory factors on END and their time course after EVT among AIS patients caused by large artery occlusion in the anterior circulation. We found that the serum concentration of IL-6 and IL-10 at admission may be a potential marker of END after EVT, and the time course of these factors is correlated with END. Additional, further larger studies are needed to confirm the current findings.

\section{Abbreviations}

END, early neurological deterioration; IL, interleukin; TNF, tumor necrosis factor; INF, interferon; BMI, body mass index; $\mathrm{DM}$, diabetes mellitus; $\mathrm{CAD}$, coronary artery disease; AF, atrial fibrillation; IS, ischemic stroke; TIA, transient ischemic attack; IQR, interquartile range; mTICI, modified Thrombolysis in Cerebral Infarction Score; NIHSS, National Institute of Health Stroke Scale; LAA, large artery atherosclerosis; TC, total cholesterol; TG, triglyceride; LDL-C, low-density lipoprotein cholesterol; HDL-C, high-density lipoprotein cholesterol; hs-CRP, high-sensitivity C-reactive protein.

\section{Data Sharing Statement}

All data supporting our results are available from the corresponding authors upon reasonable.

\section{Funding}

This work was supported by the National Natural Science Foundation of China [No. 81901215 (to Qi-Wen Deng) 
and No. 81802093 (to Hui-Ling Sun)], the Medjaden Academy \& Research Foundation for Young Scientists [No. MJR20201129 (to Qi-Wen Deng)], the Science and Technology Development Plan of Nanjing Foundation [No. 201727004 (to Hong-Chao Shi) and the Nanjing Medical Science and Technique Development Foundation Project [No. YKK18097 (to Feng Zhou)].

\section{Disclosure}

The authors declare that they have no competing interests.

\section{References}

1. Hankey GJ. Stroke. Lancet. 2017;389(10069):641-654. doi:10.1016/ S0140-6736(16)30962-X

2. Deng QW, Liu YK, Zhang YQ, et al. Low triglyceride to high-density lipoprotein cholesterol ratio predicts hemorrhagic transformation in large atherosclerotic infarction of acute ischemic stroke. Aging. 2019;11(5):1589-1601. doi:10.18632/aging.101859

3. Deng QW, Li S, Wang H, et al. The short-term prognostic value of the triglyceride-to-high-density lipoprotein cholesterol ratio in acute ischemic stroke. Aging Dis. 2018;9(3):498-506. doi:10.14336/ AD.2017.0629

4. Deng QW, Wang H, Sun CZ, et al. Triglyceride to high-density lipoprotein cholesterol ratio predicts worse outcomes after acute ischaemic stroke. Eur J Neurol. 2017;24(2):283-291. doi:10.1111/ ene. 13198

5. Byblow WD, Stinear CM. It is difficult to make predictions, especially about the future. Stroke. 2017;48(12):3187-3188. doi:10.1161/ STROKEAHA.117.019071

6. Chamorro A, Urra X, Planas AM. Infection after acute ischemic stroke: a manifestation of brain-induced immunodepression. Stroke. 2007;38(3):1097-1103. doi:10.1161/01.STR.0000258346.68966.9d

7. Deng QW, Yang H, Yan FL, et al. Blocking sympathetic nervous system reverses partially stroke-induced immunosuppression but does not aggravate functional outcome after experimental stroke in rats. Neurochem Res. 2016;41(8):1877-1886. doi:10.1007/s11064-016-1899-8

8. Schuppner R, Dirks M, Grosse GM, et al. ADAMTS-13 activity predicts outcome in acute ischaemic stroke patients undergoing endovascular treatment. Thromb Haemost. 2018;118(4):758-767.

9. Bustamante A, Sobrino T, Giralt D, et al. Prognostic value of blood interleukin- 6 in the prediction of functional outcome after stroke: a systematic review and meta-analysis. J Neuroimmunol. 2014;274 (1-2):215-224. doi:10.1016/j.jneuroim.2014.07.015

10. Denes A, Thornton P, Rothwell NJ, Allan SM. Inflammation and brain injury: acute cerebral ischaemia, peripheral and central inflammation. Brain Behav Immun. 2010;24(5):708-723. doi:10.1016/j.bbi.2009.09.010

11. Reaux-le Goazigo A, Van Steenwinckel J, Rostene W, Melik Parsadaniantz S. Current status of chemokines in the adult CNS. Prog Neurobiol. 2013;104:67-92.

12. Li X, Lin S, Chen X, et al. The prognostic value of serum cytokines in patients with acute ischemic stroke. Aging Dis. 2019;10 (3):544-556. doi:10.14336/AD.2018.0820

13. Li S, Lu G, Wang D, et al. MicroRNA-4443 regulates monocyte activation by targeting tumor necrosis factor receptor associated factor 4 in stroke-induced immunosuppression. Eur $J$ Neurol. 2020;27(8):1625-1637.

14. Suzuki S, Tanaka K, Suzuki N. Ambivalent aspects of interleukin-6 in cerebral ischemia: inflammatory versus neurotrophic aspects. J Cereb Blood Flow Metab. 2009;29(3):464-479. doi:10.1038/ jcbfm.2008.141
15. Feuerstein GZ, Wang X, Barone FC. The role of cytokines in the neuropathology of stroke and neurotrauma. Neuroimmunomodulation. 1998;5(3-4):143-159. doi:10.1159/ 000026331

16. Seners P, Hurford R, Tisserand M, et al. Is unexplained early neurological deterioration after intravenous thrombolysis associated with thrombus extension? Stroke. 2017;48(2):348-352. doi:10.1161/ STROKEAHA.116.015414

17. Adams HP Jr., Bendixen BH, Kappelle LJ, et al. Classification of subtype of acute ischemic stroke. Definitions for use in a multicenter clinical trial. TOAST. Trial of Org 10172 in Acute Stroke Treatment. Stroke. 1993;24(1):35-41. doi:10.1161/01.STR.24.1.35

18. Chamorro A, Hallenbeck J. The harms and benefits of inflammatory and immune responses in vascular disease. Stroke. 2006;37 (2):291-293. doi:10.1161/01.STR.0000200561.69611.f8

19. Esenwa CC, Elkind MS. Inflammatory risk factors, biomarkers and associated therapy in ischaemic stroke. Nat Rev Neurol. 2016;12 (10):594-604. doi:10.1038/nrneurol.2016.125

20. Chamorro A, Amaro S, Vargas M, et al. Catecholamines, infection, and death in acute ischemic stroke. J Neurol Sci. 2007;252(1):29-35. doi:10.1016/j.jns.2006.10.001

21. Kim JM, Bae JH, Park KY, et al. Incidence and mechanism of early neurological deterioration after endovascular thrombectomy. J Neurol. 2019;266(3):609-615. doi:10.1007/s00415-018-09173-0

22. Dziedzic T, Slowik A, Szczudlik A. Interleukin-6 and stroke: cerebral ischemia versus nonspecific factors influencing interleukin-6.. Stroke. 2003;34(12):e229-e230. doi:10.1161/01.STR.0000103350.88094.5B

23. Acalovschi D, Wiest T, Hartmann M, et al. Multiple levels of regulation of the interleukin-6 system in stroke. Stroke. 2003;34 (8):1864-1869. doi:10.1161/01.STR.0000079815.38626.44

24. Park SY, Kim J, Kim OJ, et al. Predictive value of circulating interleukin- 6 and heart-type fatty acid binding protein for three months clinical outcome in acute cerebral infarction: multiple blood markers profiling study. Critical Care. 2013;17(2):R45. doi:10.1186/ cc12564

25. Mechtouff L, Bochaton T, Paccalet A, et al. Association of Interleukin-6 levels and futile reperfusion after mechanical thrombectomy. Neurology. 2020;96(5):e752-e757. doi:10.1212/ WNL.0000000000011268

26. Hotter B, Hoffmann S, Ulm L, Meisel C, Fiebach JB, Meisel A. IL-6 plasma levels correlate with cerebral perfusion deficits and infarct sizes in stroke patients without associated infections. Front Neurol. 2019;10:83. doi:10.3389/fneur.2019.00083

27. Jenny NS, Callas PW, Judd SE, et al. Inflammatory cytokines and ischemic stroke risk: the REGARDS cohort. Neurology. 2019;92(20): e2375-e2384. doi:10.1212/WNL.0000000000007416

28. Sotgiu S, Zanda B, Marchetti B, et al. Inflammatory biomarkers in blood of patients with acute brain ischemia. Eur J Neurol. 2006;13 (5):505-513. doi:10.1111/j.1468-1331.2006.01280.x

29. Perini F, Morra M, Alecci M, Galloni E, Marchi M, Toso V. Temporal profile of serum anti-inflammatory and pro-inflammatory interleukins in acute ischemic stroke patients. Neurol Sci. 2001;22(4):289-296. doi:10.1007/s10072-001-8170-y

30. Hoffmann S, Harms H, Ulm L, et al. Stroke-induced immunodepression and dysphagia independently predict stroke-associated pneumonia - The PREDICT study. J Cereb Blood Flow Metab. 2017;37 (12):3671-3682. doi:10.1177/0271678X16671964

31. Mazzotta G, Sarchielli P, Caso V, et al. Different cytokine levels in thrombolysis patients as predictors for clinical outcome. Eur J Neurol. 2004;11(6):377-381. doi:10.1111/j.1468-1331.2004.00798.x

32. Smith CJ, Emsley HC, Gavin CM, et al. Peak plasma interleukin-6 and other peripheral markers of inflammation in the first week of ischaemic stroke correlate with brain infarct volume, stroke severity and long-term outcome. BMC Neurol. 2004;4(1):2. doi:10.1186/ 1471-2377-4-2 
33. Kwilasz AJ, Grace PM, Serbedzija P, Maier SF, Watkins LR. The therapeutic potential of interleukin-10 in neuroimmune diseases. Neuropharmacology. $\quad 2015 ; 96(\mathrm{Pt} \quad$ A):55-69. doi:10.1016/j. neuropharm.2014.10.020

34. Liesz A, Bauer A, Hoheisel JD, Veltkamp R. Intracerebral interleukin-10 injection modulates post-ischemic neuroinflammation: an experimental microarray study. Neurosci Lett. 2014;579:18-23. doi:10.1016/j.neulet.2014.07.003

35. Protti GG, Gagliardi RJ, Forte WC, Sprovieri SR. Interleukin-10 may protect against progressing injury during the acute phase of ischemic stroke. Arq Neuropsiquiatr. 2013;71(11):846-851. doi:10.1590/0004282X20130168

36. Singh HV, Pandey A, Shrivastava AK, Raizada A, Singh SK, Singh N. Prognostic value of neuron specific enolase and IL-10 in ischemic stroke and its correlation with degree of neurological deficit. Clin Chim Acta. 2013;419:136-138. doi:10.1016/j.cca.2013.02.014

37. van Exel E, Gussekloo J, de Craen AJ, Bootsma-van der Wiel A, Frolich M, Westendorp RG. Inflammation and stroke: the Leiden 85-Plus Study. Stroke. 2002;33(4):1135-1138. doi:10.1161/01. STR.0000014206.05597.9E
38. Xie G, Myint PK, Zaman MJ, et al. Relationship of serum interleukin-10 and its genetic variations with ischemic stroke in a Chinese general population. PLoS One. 2013;8(9):e74126. doi:10.1371/journal.pone.0074126

39. Li S, Lu G, Wang D, et al. MicroRNA-4443 regulates monocyte activation by targeting TRAF4 in stroke-induced immunosuppression. Eur J Neurol. 2020;27(8):1625-1637. doi:10.1111/ene.14282

40. Wang H, Deng QW, Peng AN, et al. beta-arrestin2 functions as a key regulator in the sympathetic-triggered immunodepression after stroke. J Neuroinflammation. 2018;15(1):102. doi:10.1186/s12974018-1142-4

41. Xiong X, Barreto GE, Xu L, Ouyang YB, Xie X, Giffard RG. Increased brain injury and worsened neurological outcome in interleukin-4 knockout mice after transient focal cerebral ischemia. Stroke. 2011;42 (7):2026-2032. doi:10.1161/STROKEAHA.110.593772

42. Pena-Philippides JC, Caballero-Garrido E, Lordkipanidze T, Roitbak T. In vivo inhibition of miR-155 significantly alters post-stroke inflammatory response. J Neuroinflammation. 2016;13 (1):287. doi:10.1186/s12974-016-0753-x
Journal of Inflammation Research

\section{Publish your work in this journal}

The Journal of Inflammation Research is an international, peerreviewed open-access journal that welcomes laboratory and clinical findings on the molecular basis, cell biology and pharmacology of inflammation including original research, reviews, symposium reports, hypothesis formation and commentaries on: acute/chronic inflammation; mediators of inflammation; cellular processes; molecular

\section{Dovepress}

mechanisms; pharmacology and novel anti-inflammatory drugs; clinical conditions involving inflammation. The manuscript management system is completely online and includes a very quick and fair peerreview system. Visit http://www.dovepress.com/testimonials.php to read real quotes from published authors. 